\title{
A Consistent Scenario for the IBEX Ribbon, Anisotropies in TeV Cosmic Rays, and the Local Interstellar Medium
}

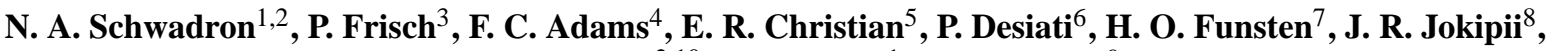 \\ D. J. McComas ${ }^{2,10}$, E. Moebius ${ }^{1}$, and G. Zank ${ }^{9}$ \\ ${ }^{1}$ University of New Hampshire, Durham, NH, 03824, UK \\ ${ }^{2}$ Southwest Research Institute, San Antonio, TX 78228, USA \\ ${ }^{3}$ University of Chicago, Department of Astronomy and Astrophysics, Chicago, IL 60637, USA \\ ${ }^{4}$ University of Michigan, Ann Arbor, MI, 48109, USA \\ ${ }^{5}$ Goddard Space Flight Center, Greenbelt, MD, 20771 \\ ${ }^{6}$ IceCube Research Center and Astronomy Department, University of Wisconsin, Madison, WI 53706, USA \\ ${ }^{7}$ Los Alamos National Laboratory, Los Alamos, NM, 87545, USA \\ ${ }^{8}$ University of Arizona, Tucson, AX 85721, USA \\ ${ }^{9}$ University of Alabama, Huntsville, AL, Huntsville, AL 35899, USA \\ ${ }^{10}$ University of Texas at San Antonio, San Antonio, TX, USA \\ Correspondence to: N. A. Schwadron (n.schwadron@unh.edu)
}

Received: 2 April 2015 - Revised: 28 July 2015 - Accepted: 24 August 2015 - Published: 8 September 2015

\begin{abstract}
The Interstellar Boundary Explorer (IBEX) observes enhanced $\sim \mathrm{keV}$ energy Energetic Neutral Atoms (ENAs) from a narrow "ribbon" that stretches across the sky and appears to be centered on the direction of the local interstellar magnetic field. The Milagro collaboration, the As $\gamma$ collaboration and the IceCube observatory have made global maps of $\mathrm{TeV}$ cosmic rays. This paper provides links between these disparate observations. We develop a simple diffusive model of the propagation of cosmic rays and the associated cosmic ray anisotropy due to cosmic ray streaming against the local interstellar flow. We show that the local plasma and field conditions sampled by IBEX provide characteristics that consistently explain $\mathrm{TeV}$ cosmic ray anisotropies. These results support models that place the interstellar magnetic field direction near the center of the IBEX ribbon.
\end{abstract}

\section{Introduction}

The Interstellar Boundary Explorer Mission (IBEX), launched October 2008, has the objective to discover the global interaction between the solar wind and the local interstellar medium (McComas et al., 2009b). IBEX measures from $\sim 0.01$ to $\sim 6 \mathrm{keV}$ neutral atoms. In the $\sim \mathrm{keV}$ energy range these particles, dubbed energetic neutral atoms (ENAs), are created predominantly from charge-exchange between neutral interstellar hydrogen and plasma protons in the solar wind and interstellar medium (McComas et al., 2009b, 2011). IBEX also makes measurements of lower en- ergy atoms from the interstellar medium including oxygen, helium and hydrogen atoms (Möbius et al., 2009).

The IBEX maps revealed the existence of a narrow ribbon of higher flux ENA emissions (McComas et al., 2009a; Funsten et al., 2009; Fuselier et al., 2009). The ribbon forms a roughly circular structure centered on (gal. long $\ell$, gal. lat. $b$ ) $\sim\left(210.5^{\circ} \pm 2.6^{\circ},-57.1^{\circ} \pm 1.0\right)$, with the center of the ribbon arc varying by $\sim 11^{\circ}$ over the measured energy range (Funsten et al., 2013). The center of the IBEX ribbon likely defines the direction of the local interstellar (LISM) magnetic field (Schwadron et al., 2009). However, until the physical mechanism for the ribbon is identified, it will be difficult to know how the interstellar field direction is related in 
detail to the IBEX ribbon. A number of heliospheric models have been devised with the property that the ribbon may be highly sensitive to the interstellar magnetic field direction (Heerikhuisen et al., 2010, 2014; Frisch et al., 2010; Ratkiewicz et al., 2012; Schwadron and McComas, 2013). For the purposes of this paper, we take the association of the IBEX ribbon center with the interstellar magnetic field direction as a partially-supported conjecture, and attempt to build a consistent scenario for the $\mathrm{TeV}$ cosmic ray anisotropies. Further, this study supplies a test for the association between the IBEX ribbon and the interstellar magnetic field. This paper also discusses another observational signature of this LISM magnetic field in $\mathrm{TeV}$ cosmic ray anisotropies (Schwadron et al., 2014).

There are numerous studies that complement the analysis presented here. While Schwadron et al. (2014) studied the global $\mathrm{TeV}$ anisotropy, there are also features in $\mathrm{TeV}$ cosmic ray maps that may be directly related to heliospheric structure. For example, Desiati and Lazarian (2013) considered the scenario where heliospheric magnetic instabilities (see Pogorelov et al., 2013) cause resonant scattering which may re-distribute the anisotropic cosmic rays in the 10 's $\mathrm{TeV}$ energy scale. Such scattering processes may produce a complex angular structure in the arrival direction distribution of cosmic rays, that trace the heliospheric structure. Another complementary analysis is presented by Effenberger et al. (2012), which examines Galactic transport models for cosmic rays involving the diffusive motion of these particles in the interstellar medium. Because of the large-scale structure of the Galactic magnetic field, cosmic ray diffusion is found to be highly anisotropic. As a result, significant variability is found in the energy spectra at different positions along the Sun's Galactic orbit. Locally, polarized starlight and the center of the IBEX ribbon arc trace the same interstellar field directions to within uncertainties, suggesting that the ordering of the interstellar field persists over much larger spatial scales than that of the heliosphere (Frisch et al., 2015). These results highlight the need to account for anisotropic diffusion and the need for accurate Galactic magnetic field models. The work presented here offers new insights into local structure of the magnetic field in the interstellar medium.

\section{Interstellar flow and field characteristics}

Our understanding of the characteristics of the local interstellar medium (LISM) depend critically on a limited set of observations. Very recent analysis of Interstellar Boundary Explorer observations has allowed us to update estimates of LISM flow using observations of interstellar neutral (ISN) He (e.g., McComas et al., 2015b, a; Schwadron et al., 2015; Bzowski et al., 2015; Leonard et al., 2015; Möbius et al., 2015). A feature of the IBEX data is that the LIC physical characteristics are determined from a narrow tube in parameter space where the interstellar parameters are approx- imately degenerate. However, analysis resulting in a specific solution was associated with large uncertainties along the parameter tube. The more recent IBEX analyses have taken into account a much larger observational baseline, allowing derivation of specific solutions. In the Heliocentric coordinates, Schwadron et al. (2015) found an ISN flow gal. long. $\ell=183.7^{\circ} \pm 0.8^{\circ}$, gal. lat. $b=-15.0^{\circ} \pm 1.5^{\circ}$, and speed $25.4 \pm 1.1 \mathrm{~km} \mathrm{~s}^{-1}$. This yields an angle of $80.4^{\circ} \pm 5^{\circ}$ between the magnetic field and the velocity expressed with respect to the LSR velocity frame that describes our galactic neighborhood. This angle is $46.6^{\circ} \pm 2.5^{\circ}$ for velocities given with respect to the Sun.

This yields an angle between that magnetic field and the flow velocity in the LSR frame of $80.4^{\circ} \pm 5^{\circ}$. This angle is $46.6^{\circ} \pm 2.5^{\circ}$ in the Heliocentric reference frame.

\section{Local Interstellar Structure}

The heliosphere interacts with a clumpy decelerating interstellar flow that moves away from the Lower Centaurus-Crux (LCC) association near the origin of the Loop I superbubble (Frisch et al., 2011). The Sun is likely located within or near the Local Interstellar Cloud (LIC), less than $0.1 \mathrm{pc}$ from the LIC edge (Wood et al., 2000; Frisch and Mueller, 2011). In the direction facing away from the galactic center, the closest cloud to the LIC is the "Blue Cloud" (BC, Lallement et al., 1994; Hébrard et al., 1999). The conditions for colliding clouds are observed within $\sim 10 \mathrm{pc}$ (Linsky et al., 2008), and relative intercloud velocities are observed up to $50 \mathrm{~km} \mathrm{~s}^{-1}$. Gry and Jenkins (2015) model the LIC kinematics by omitting outlying velocity components, and conclude that a shock front is driving into the cloud from the rough location of $\ell=124^{\circ}, b=-67^{\circ}$ (or RA, DEC $=13^{\circ},-4^{\circ}$ ). Figure 1 provides a rough picture of local interstellar structure near the edge of LIC and at the front of the Loop I superbubble.

An interstellar magnetic field entrained in the inhomogeneous interstellar material associated with the expanding Loop I shell provides an explanation for inhomogeneities in the local interstellar magnetic field. Measurements by IBEX and Ulysses of interstellar He inside of the heliosphere provide the LIC velocity (e.g., McComas et al., 2015b, a; Schwadron et al., 2015; Bzowski et al., 2015; Leonard et al., 2015; Möbius et al., 2015) and velocities for 15 local clouds have been determined with the triangulation of the radial velocity component towards different stars behind the clouds (Redfield and Linsky, 2008). The Loop I superbubble shell is centered $\approx 78 \mathrm{pc}$ at galactic coordinates of $\ell, b \approx 346^{\circ}$, $3^{\circ}$ (Wolleben, 2007). Pressure equilibrium between the gas and magnetic field in the LIC yield magnetic field strength of $\sim 2.7 \mu \mathrm{G}$ (Slavin and Frisch, 2008). This value is similar to the $\sim 3 \mu \mathrm{G}$ field strength derived from the globally distributed ENA fluxes (Schwadron et al., 2011).

The IBEX magnetic field direction should be apparent in the light of nearby stars that is linearly polarized in the inter- 


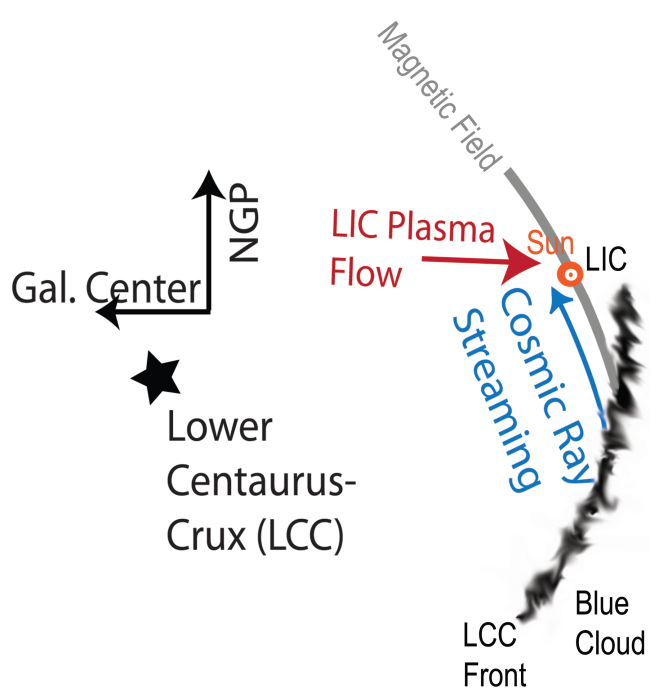

Figure 1. Sketch of the local interstellar environment projected in the plane containing the vectors to the galactic center and to the north galactic pole (NGP). The grey curve shows the magnetic field connection from the heliosphere to the front, which provides access of cosmic rays (blue arrow) to the heliosphere. The red arrow shows the direction of the local interstellar flow. We also show rough locations of the LIC and the Blue Cloud, which exist in a region near the front from the LCC where the conditions exist for intercloud collisions.

stellar medium. The spatial region of the LIC, as traced by stars with interstellar absorption lines at the LIC velocity, is shown in Fig. 2 (open circles). Comparisons between Fig. 2 and Fig. 4 show that the regions with the highest $\mathrm{TeV}$ cosmic ray intensities are also the regions where the LIC is located.

Nearby stars with polarizations that best agree with IBEX Interstellar Magnetic Field (ISMF) direction are plotted as red dots in Fig. 2; these data for stars within $90^{\circ}$ of the heliosphere nose give a magnetic field direction toward $\ell, b=36^{\circ}$, $49^{\circ}, \pm 16^{\circ}$ (Frisch et al., 2015). Figure 2 also shows stars with polarization data that do not sample the IBEX ISMF, either because the polarization vectors point in another direction or the polarizations are insignificant (small black dots). The similar locations of the polarizations that best-trace the ISMF direction and the LIC stars suggests that the interstellar magnetic field direction traced by the center of the IBEX ribbon corresponds to magnetic field lines within the LIC.

Taken together, these results suggest that the magnetic field inside the rim of the Loop I superbubble at the solar location is associated with the LIC and is consistent with the magnetic field that orders the IBEX Ribbon (also see Frisch et al., 2015).

\section{Source of Large-scale anisotropy in TeV cosmic rays}

We consider a $\mathrm{TeV}$ cosmic ray model for the large-scale anisotropy associated with streaming of cosmic rays against the LIC plasma flow. We stress that the anisotropy reported by the IceCube, the Milagro and As $\gamma$ collaborations have been corrected for Earth's motion. In practice, the TeV cosmic ray anisotropy maps represent the flux distribution in an inertial reference frame moving with the Sun. Because TeV cosmic rays have large gyro-radii, on the order of the size of the heliosphere, the $\mathrm{TeV}$ anisotropy maps are characterized by the cosmic ray velocity distribution incident from outside the heliosphere, and are affected by interactions of cosmic rays with the heliosphere (Schwadron et al., 2014).

We break up the calculation of the anisotropy into two pieces: (1) we use standard diffusion theory to determine the anisotropy incident from beyond the heliosphere; (2) we characterize the interaction of $\mathrm{TeV}$ cosmic rays with the heliosphere and solve for the resulting distortion of $\mathrm{TeV}$ cosmic ray anisotropies. Both of these calculations are worked in detail by Schwadron et al. (2014). In particular, it was shown the the heliosphere makes small changes to the global anisotropy at energies greater than $10 \mathrm{TeV}$. In the present work, we focus on the calculation of the global anisotropy incident on the global heliosphere. This calculation of the $\mathrm{TeV}$ cosmic ray anisotropy is an idealization that essentially neglects the presence of the heliosphere. The calculation is most important in showing how the local interstellar magnetic field and plasma conditions control the overall magnitude and an orientation of $\mathrm{TeV}$ cosmic ray anisotropies.

Standard diffusion theory (e.g., Forman and Gleeson, 1975) specifies the anisotropic component of the distribution in cosmic rays,

$\xi=\frac{3}{w}\left[C \boldsymbol{u}-\kappa_{\|} \mathbf{g}_{\|}-\kappa_{\perp} \mathbf{g}_{\perp}-\kappa_{T}(\mathbf{g} \times \hat{\boldsymbol{b}})\right]$.

The anisotropy is related to the streaming $\mathbf{S}$ and the isotropic component $f_{0}$ of the cosmic ray distribution, $\boldsymbol{\xi}=$ $3 \mathbf{S} /\left(4 \pi w f_{0}\right)$. The streaming is $\mathbf{S}=\int \mathrm{d} \Omega \mathbf{w} f$, where $\mathbf{w}$ is the velocity of cosmic rays, and $\int \mathrm{d} \Omega$ indicates integration over solid-angle. The diffusion coefficients $\kappa_{\|}$and $\kappa_{\perp}$ are the parallel and perpendicular to the magnetic field. The bulk velocity of the LISM plasma is $\boldsymbol{u}$ and the term $\mathbf{g}=\nabla \ln \left(f_{0}\right)$ is the gradient of the isotropic density. The quantity $\mathbf{g}_{\|}=(\hat{\boldsymbol{b}} \cdot \mathbf{g}) \hat{\boldsymbol{b}}$ is the parallel component of the gradient, and $\mathbf{g}_{\perp}$ is the perpendicular component. The term $\kappa_{T}=\Omega \tau \kappa_{\perp}$ is the off-diagonal diffusion coefficient, $\tau$ is the scattering time, and $\Omega$ is the gyrofrequency. The Compton-Getting coefficient is defined as follows: $C=(-1 / 3)\left[\partial \ln f_{0} / \partial \ln p\right]$. 


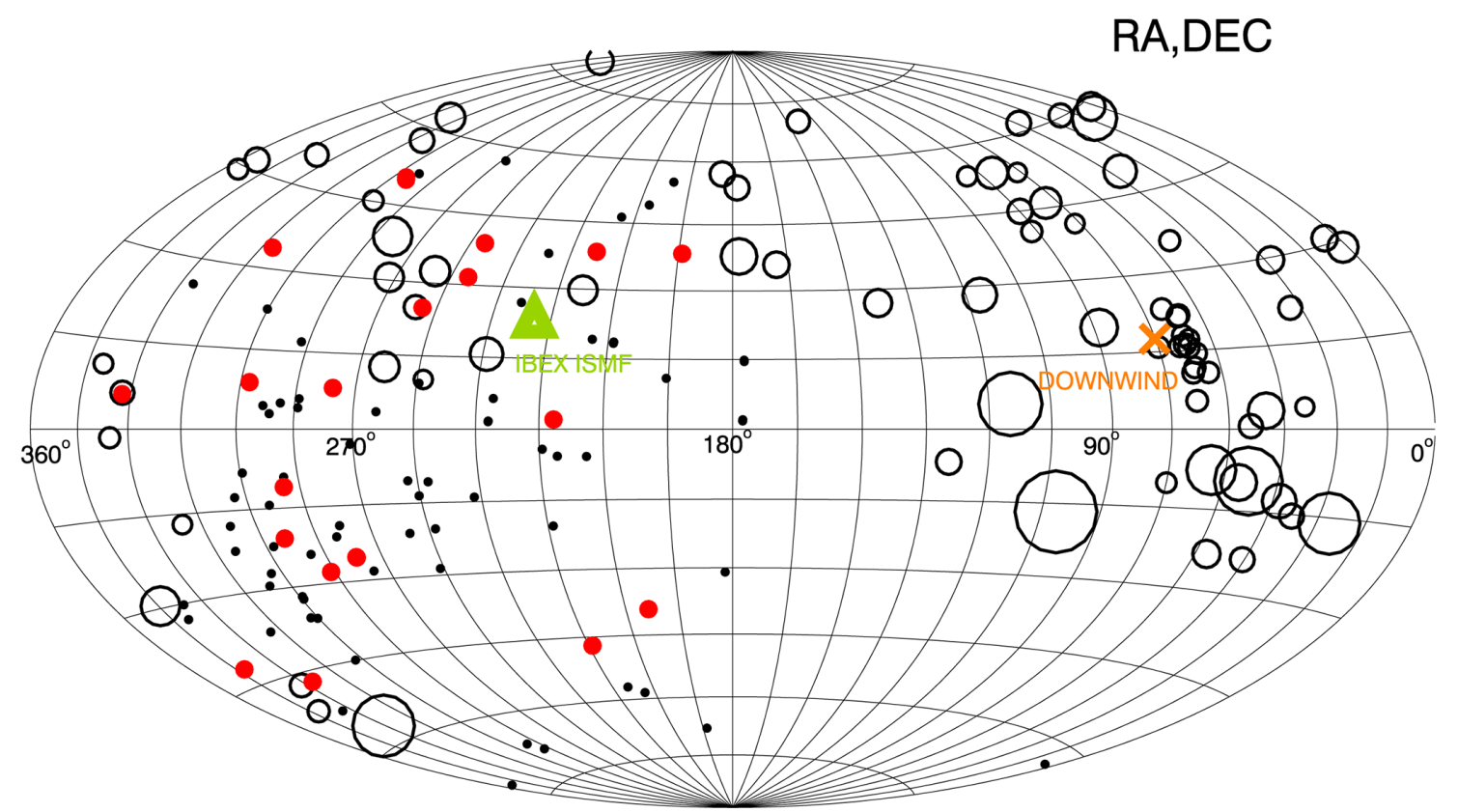

Figure 2. The locations of stars that trace the LIC are plotted in right ascension and declination (open black circles), based on the description of the LIC in the 15-cloud model of Redfield and Linsky (2008). The size of the LIC symbol is inversely proportional to the star distance. Small black dots indicate stars with polarization data collected in the 21 st century and within $90^{\circ}$ of the heliosphere nose (from Frisch et al., 2015). The red dots indicate the half of the group of significant polarization position angles that are in best agreement with the dominant ISMF direction near the Sun. Since the dominant ISMF is the same as the IBEX ISMF direction to within the uncertainties, the red dots also trace the extension of the IBEX magnetic field out into interstellar space for the sampled region. Comparisons between the LIC stars and the GCR asymmetries in Fig. 4 show that higher GCR fluxes tend to be associated with sightlines through the LIC, where the red dots show that the IBEX ISMF is also found. Locations are plotted in equatorial coordinates.

The full distribution function is related to the isotropic distribution and the anisotropy,

$f(\mathbf{p})=f_{0}(p) \times(1+\boldsymbol{\xi} \cdot \hat{\boldsymbol{p}})$.

The unit vector $\hat{\boldsymbol{p}}$ is in the direction of the cosmic ray momentum. Based on this formulation, a solution of the isotropic portion of distribution function, $f_{0}$, including its spatial gradients allows specification of the anisotropy.

The needed solution for the isotropic portion of the distribution function $f_{0}$ is found from the Parker transport equation,

$$
\frac{\partial f_{0}}{\partial t}+\boldsymbol{u} \cdot \nabla f_{0}-\nabla \cdot\left(\mathbf{K} \cdot \nabla f_{0}\right)-\frac{\nabla \cdot \boldsymbol{u}}{3} p \frac{\partial f_{0}}{\partial p}=0,
$$

where $\mathbf{K}$ is the diffusion tensor. The differential energy spectrum is taken as power-law form of $j \propto T^{-2.7}$ where $T$ is kinetic energy. In this case, the distribution function has the power-law, $f_{0} \propto p^{-4.7}$, and $C=4.7 / 3=1.6$

The anisotropy can be obtained from Eq. (1) provided that we have information about the spatial dependence of the distribution function. We take the Local Standard of Rest (LSR) frame where the distribution is scattered toward isotropy. In this frame, we consider the relative plasma flow, $u_{\mathrm{LSR}}$, directed at angle $\theta$ relative to the magnetic field. As discussed in Sect. 1 and Table 1, we utilize the local determinations from IBEX for the interstellar plasma flow and the local magnetic field from the IBEX ribbon as proxies for these vectors on the larger parsec-scales needed needed to specify the cosmic ray anisotropy magnitude (Jokipii, 2005, 2007). Note that the local flow measured by IBEX, $\boldsymbol{u}_{\text {LISM }}$, is added to the solar apex motion, $\boldsymbol{u}_{\odot}$, to determine the flow in the LSR frame, $\boldsymbol{u}_{\mathrm{LSR}}$ :

$\boldsymbol{u}_{\mathrm{LSR}}=\boldsymbol{u}_{\odot}+u_{\mathrm{LISM}}$.

The Parker transport equation is written as

$\frac{\partial}{\partial x}\left[\boldsymbol{u}_{\mathrm{LSR}} f_{0}-\kappa \frac{\partial f_{0}}{\partial x}\right]=0$.

In this case, the $x$ axis is directed along $\boldsymbol{u}_{\mathrm{LSR}}$ and $\kappa=$ $\kappa_{\|} \cos ^{2} \theta+\kappa_{\perp} \sin ^{2} \theta$ is the diffusion tensor projected along the direction of the plasma flow. The solution upstream is

$f_{0} \propto \exp \left(\frac{u_{\mathrm{LSR}}}{\kappa_{x x}} x\right)$

and $f_{0} \propto$ constant downstream. Equation (6) is an approximation in the limit of 1-D uniform flow and a planar shock geometry. Implications for $\mathrm{TeV}$ cosmic ray anisotropies of 
Table 1. Local interstellar parameters.

\begin{tabular}{|c|c|c|c|}
\hline & $\begin{array}{c}\text { Mag. } \\
\left(\mathrm{kms}^{-1}\right)\end{array}$ & $\begin{array}{c}\text { Gal. } \\
\text { Long. } \ell\left(^{\circ}\right)\end{array}$ & $\begin{array}{c}\text { Gal. } \\
\text { Lat. } b\left(^{\circ}\right)\end{array}$ \\
\hline LISM (HC frame $\left.{ }^{\mathrm{a}}\right), \boldsymbol{u}_{\text {LISM }}$ & $25.4 \pm 1.1$ & $183.7 \pm 0.8$ & $-15.0 \pm 1.5$ \\
\hline Solar apex motion in $\mathrm{LSR}^{\mathrm{b}}, \boldsymbol{u}_{\odot}$ & $18.0 \pm 0.9$ & $47.8 \pm 2.9$ & $23.8 \pm 2.0$ \\
\hline LISM (LSR frame), $\boldsymbol{u}_{\mathrm{LSR}}$ & $17.2 \pm 1.1$ & $141.6 \pm 3.7$ & $2.3 \pm 2.9$ \\
\hline LISM Mag. Field ${ }^{\mathrm{c}}$ & & $210.5 \pm 2.6$ & $-57.1 \pm 1.0$ \\
\hline
\end{tabular}

${ }^{a}$ From Schwadron et al. (2015) and in Heliocentric rest frame.

b Solar apex motion in Local Standard of Rest, LSR (Schönrich et al., 2010).

${ }^{c}$ Field direction taken from the IBEX highest energy steps (1.79 and $2.73 \mathrm{keV}$, Funsten et al., 2013)

which shows ribbon coherence and a long Line-of-Sight. hello world.

departures from this approximation should be explored in the future. The use of the LSR frame to calculate the cosmic ray gradients since the gradient scale, $\kappa_{x x} / u_{\mathrm{LSR}}$, is typically more than 1000's of parsecs at TeV energies.

We apply the upstream solution because the flow is a partof the Loop I superbubble expansion. The anisotropy is given by

$$
\begin{aligned}
\boldsymbol{\xi}= & \frac{3}{w}\left[C \boldsymbol{u}_{\mathrm{LSR}}-\frac{\kappa_{\|}}{\kappa_{x x}} u_{\mathrm{LSR}} \cos \theta \hat{\boldsymbol{b}}-\right. \\
& \left.\frac{\kappa_{\perp}}{\kappa_{x x}} u_{\mathrm{LSR}} \sin \theta \hat{\boldsymbol{e}}_{\perp 1}-\frac{\kappa_{T}}{\kappa_{x x}} u_{\mathrm{LSR}} \sin \theta \hat{\boldsymbol{e}}_{\perp 2}\right]
\end{aligned}
$$

and the unit vectors $\hat{\boldsymbol{e}}_{\perp 1}=[\hat{\boldsymbol{x}}-\cos (\theta) \hat{\boldsymbol{b}}] / \sin (\theta)$ and $\hat{\boldsymbol{e}}_{\perp 2}=$ $[\hat{\boldsymbol{x}} \times \hat{\boldsymbol{b}}] / \sin (\theta)$ are orthonormal. The components $\hat{\boldsymbol{b}}, \hat{\boldsymbol{e}}_{\perp 1}$, and $\hat{\boldsymbol{e}}_{\perp 2}$ form a basis with projections of associated with parallel diffusion $(\hat{\boldsymbol{b}})$, perpendicular diffusion $\left(\hat{\boldsymbol{e}}_{\perp 1}\right)$, and drift $\left(\hat{\boldsymbol{e}}_{\perp 2}\right)$.

As a simple example, we adopt the parallel diffusion coefficient (Büsching and Potgieter, 2008; Ptuskin et al., 2006)

$\kappa_{\|}=\kappa_{0}\left(\zeta / \zeta_{0}\right)^{0.6}$

where $\zeta=p c / q$ is rigidity $q$ is the cosmic-ray charge, and $\kappa_{0}=0.073(\mathrm{kpc})^{2}(\mathrm{Myr})^{-1}$ for $\zeta_{0}=3 \mathrm{GV}$ (these specific values were obtained by Ptuskin et al., 2006). Figure 3 shows the resulting anisotropy broken down by component magnitude in Eq. (7) as a function of $\kappa_{\perp} / \kappa_{\|}$at $5 \mathrm{TeV}$. In this figure we have varied the field-flow angle over the region of uncertainty from 75.4 to $85.4^{\circ}$. A $\sim 0.1-0.2 \%$ anisotropy is approached in the limit of relatively small values of $\kappa_{\perp} / \kappa_{\|}$.

The resulting global anisotropy map in $\mathrm{TeV}$ cosmic rays is shown in Fig. 4. The full calculation is detailed by Schwadron et al. (2014) and includes the interaction of cosmic rays with the interstellar magnetic field deflected around the heliosphere. Notably, Schwadron et al. (2014) show that the gyroradii of $\mathrm{TeV}$ cosmic rays are on the scale or larger than the scale of the heliosphere itself. As a result, the effects of the heliosphere on TeV cosmic rays cannot be modeled using the Parker transport equation, which relies on a diffusive approximation and moment decomposition of the cosmic ray distribution. Instead, Schwadron et al. (2014) detailed the effects of the heliosphere by following individual cosmic ray trajectories and mapping the effects of the local interstellar

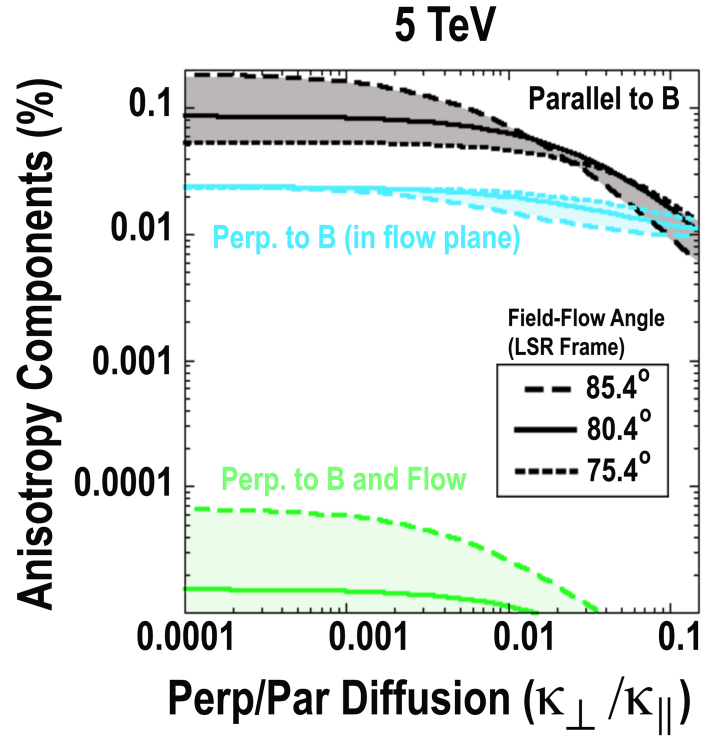

Figure 3. Components (parallel to B in black, perpendicular to B in the flow plane in blue, and perpendicular to $\mathrm{B}$ and the flow in green) of the cosmic ray anisotropy as a function of $\kappa_{\perp} / \kappa_{\|}$at $5 \mathrm{TeV}$. The field-plasma angle is varied between 75.4 and $85.4^{\circ}$. The shaded regions correspond to the regions covered by these variations.

magnetic field deflected about the heliosphere on the cosmic ray distribution function. The magnetic field structure calculated by Schwadron et al. (2014) is based on a potential approximation. Recent work by Röken et al. (2015) offers a new analytical solution for the interstellar magnetic field in the vicinity of the heliosphere. This provides an opportunity to contrast the influences of different interstellar field models near the heliosphere on the propagation of cosmic rays. The model of Schwadron et al. (2014) does not include stochastic acceleration, heating by magnetic reconnection, or interaction with the magnetic and electric fields inside the heliopause. The main free parameter in the estimates of the global $\mathrm{TeV}$ anisotropy is the level of perpendicular diffusion, for which we use a value typical for the ISM, $\kappa_{\perp} / \kappa_{\|} \sim 0.001$. This level of perpendicular diffusion is similar to that found by Shalchi et al. (2010).

It is important to note that the field-flow angle plays a significant role in determining the maximum anisotropy. In Schwadron et al. (2014), based on knowledge of the LISM at the time of publication, the field-flow angle of $87.6^{\circ}$ was used. This value is slightly above the central value plus uncertainty of $85.4^{\circ}$ cited in Table 1 . However, as shown in Fig. 3, a maximum anisotropy of $0.2 \%$ can still be achieved with a field-flow angle of $85.4^{\circ}$. A more significant issue, however, is whether the "local" value of the field-flow angle returned by IBEX is relevant over the larger pc or 10's of pc scales critical for determining the $\mathrm{TeV}$ anisotropies. Based on the agreement between simulated and observed anisotropy, the tentative conclusion is that the local deter- 


\section{Observed}

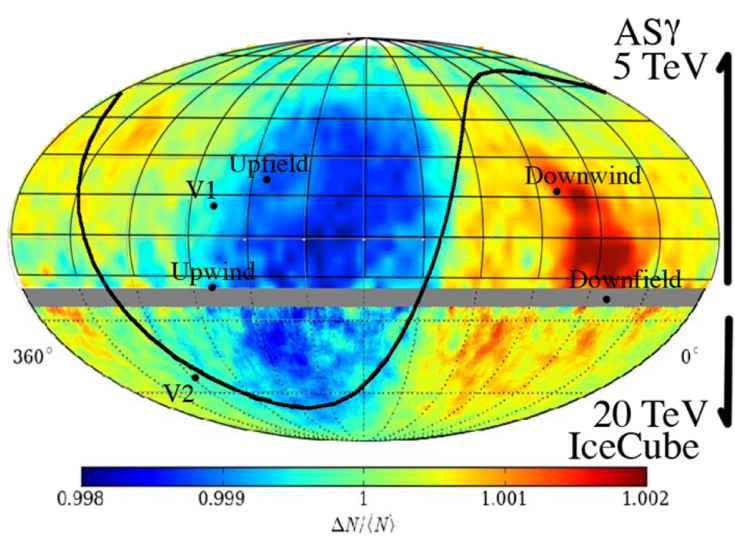

\section{Interstellar Conditions from IBEX}

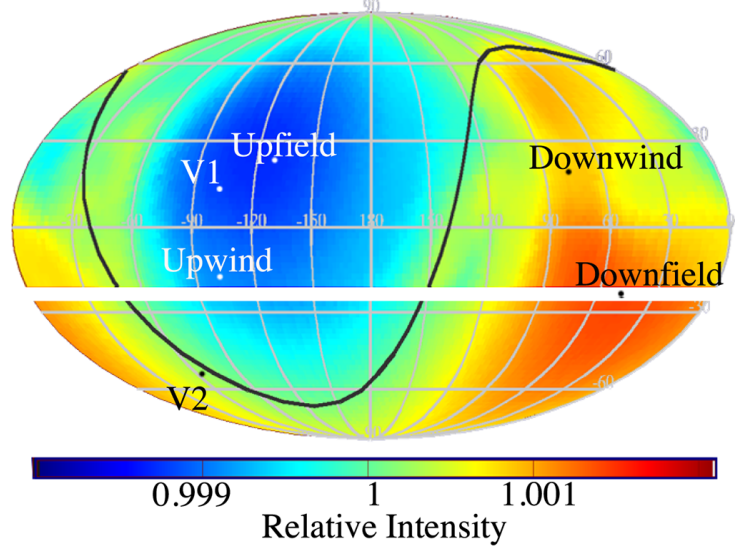

Figure 4. (Taken from Schwadron et al., 2014.) Based on the anisotropy model, we compare observed (left) and modeled (right) cosmic ray intensities. Below $25^{\circ} \mathrm{S}$ latitude we show the IceCube anisotropy map, which has a median energy of $20 \mathrm{TeV}$. On the left panel, above $20^{\circ} \mathrm{S}$ latitude, we show the anisotropy map from AS- $\gamma$ with $5 \mathrm{TeV}$ median energy. On the right panel, the modeled map at $20 \mathrm{TeV}$ is shown for latitudes below $25^{\circ} \mathrm{S}$ and at $5 \mathrm{TeV}$ for latitudes above $20^{\circ} \mathrm{S}$.

mination of the field-flow angle does appear to be applicable over much larger scales, albeit with some reasonable uncertainty due to small-scale variations in both the field and flow. The large field-flow angle, close to $90^{\circ}$, should be a natural consequence of the sweeping and subsequent wrapping up of the field at the outer edge of the Loop I superbubble expansion.

Another surprising aspect of the calculation presented here and by Schwadron et al. (2014) is that the global TeV anisotropy appears to agree in both magnitude and overall orientation based on diffusive streaming in response to advection by the local interstellar flow. As a result, the cosmic ray anisotropy is aligned with the spatial configuration of the LIC. The LIC magnetic field coincides with the IBEX magnetic field direction to within the uncertainties and is entrained in the rim of the expanding Loop I leading to streaming of the GCRs along the magnetic field swept up by the expansion of the Loop I/LCC superbubble (see also the schematic in Fig. 1).

\section{Summary}

We detail here a simple model for the $\mathrm{TeV}$ cosmic ray anisotropy based on a diffusive anisotropy created in response to advection by the local interstellar flow. The global configuration of the anisotropy is strongly ordered by the interstellar magnetic field. By assumpition, the model uses a orientation for the interstellar magnetic field taken from the center of the IBEX ribbon (see Funsten et al., 2013) and a flow direction taken from recent interstellar neutral He measurements (e.g., McComas et al., 2015b, a; Schwadron et al., 2015; Bzowski et al., 2015; Leonard et al., 2015; Möbius et al., 2015; Bzowski et al., 2014; Wood et al., 2015). The fact that model results appear to agree with the general organization of the observed $\mathrm{TeV}$ anisotropy strengthens our assumption for the interstellar field direction, and suggests that the local determinations are indicative of large-scale characteristics within the local interstellar medium.

There is a significant factor that may help create conditions conducive to aligning local and global organization of the magnetic field and flow. Our Sun's position within the LIC appears to be near the outskirts of the Loop I superbubble. At this position, the expansion of the interstellar plasma from the center of the Loop I superbubble sweeps up the interstellar magnetic field, thereby maintaining a field-flow angle close to $90^{\circ}$. This field direction is consistent with that inferred from the center of the IBEX ribbon.

Clearly the local interstellar conditions observed by the Interstellar Boundary Explorer provide fundamental insights into the magnetic and flow characteristics that order $\mathrm{TeV}$ cosmic ray anisotropies.

Acknowledgements. We are deeply indebted to all of the outstanding people who have made the IBEX mission possible and have contributed to the As $\gamma$, IceCube and Milagro projects. This work was carried out as a part of the IBEX project, with support from NASA's Explorer Program.

Edited by: K. Scherer

Reviewed by: H. Fichtner and one anonymous referee 


\section{References}

Büsching, I. and Potgieter, M. S.: The variability of the proton cosmic ray flux on the Sun's way around the galactic center, Adv. Space Res., 42, 504-509, 2008.

Bzowski, M., Kubiak, M. A., Hłond, M., Sokół, J. M., Banaszkiewicz, M., and Witte, M.: Neutral interstellar He parameters in front of the heliosphere 1994-2007, Astron. Astrophys., 569, 11 pp. doi:10.1051/0004-6361/201424127, 2014.

Bzowski, M., Swaczyna, P., Kubiak, M. A., Sokół, J. M., Moebius, E., Leonard, T., Heirtzler, D., Schwadron, N. A., Fuselier, S., Kucharek, H., Galli, A., Wurz, P., and McComas, D. J.: Impact of Planetary Gravitation on High Precision Neutral Atom Measurements, Astrophys. J. Suppl. S., in press, 2015.

Desiati, P. and Lazarian, A.: Anisotropy of TeV Cosmic Rays and Outer Heliospheric Boundaries, Astrophys. J., 762, 44, doi:10.1088/0004-637X/762/1/44, 2013.

Effenberger, F., Fichtner, H., Scherer, K., and Büsching, I.: Anisotropic diffusion of Galactic cosmic ray protons and their steady-state azimuthal distribution, Astron. Astrophys., 547, A120, doi:10.1051/0004-6361/201220203, 2012.

Forman, M. A. and Gleeson, L. J.: Cosmic-ray streaming and anisotropies, Astron. Astrophys. Suppl. S., 32, 77-94, 1975.

Frisch, P. C. and Mueller, H.-R.: Time-Variability in the Interstellar Boundary Conditions of the Heliosphere: Effect of the Solar Journey on the Galactic Cosmic Ray Flux at Earth, Space Sci. Rev., 176, 21-34, 2011.

Frisch, P. C., Andersson, B.-G., Berdyugin, A., Funsten, H. O., Magalhaes, A. M., McComas, D. J., Piirola, V., Schwadron, N. A., Slavin, J. D., and Wiktorowicz, S. J.: Comparisons of the Interstellar Magnetic Field Directions Obtained from the IBEX Ribbon and Interstellar Polarizations, Astrophys. J., 724, 1473, doi:10.1088/0004-637X/724/2/1473, 2010.

Frisch, P. C., Redfield, S., and Slavin, J. D.: The Interstellar Medium Surrounding the Sun, Annu. Rev. Astron. Astr., 49, 237-279, doi:10.1146/annurev-astro-081710-102613, 2011.

Frisch, P. C., Berdyugin, A., Piirola, V., Magalhaes, A. M., Seriacopi, D. B., Wiktorowicz, S. J., Andersson, B.-G., Funsten, H. O., McComas, D. J., Schwadron, N. A., Slavin, J. D., Hanson, A. J., and Fu, C.-W.: Charting the Interstellar Magnetic Field behind the Interstellar Boundary Explorer (IBEX) Ribbon of Energetic Neutral Atoms, Astrophys. J., in review, 2015.

Funsten, H. O., Allegrini, F., Crew, G. B., DeMajistre, R., Frisch, P. C., Fuselier, S. A., Gruntman, M., Janzen, P., McComas, D. J., Möbius, E., Randol, B., Reisenfeld, D. B., Roelof, E. C., and Schwadron, N. A.: Structures and Spectral Variations of the Outer Heliosphere in IBEX Energetic Neutral Atom Maps, Science, 326, 964-966, doi:10.1126/science.1180927, 2009.

Funsten, H. O., DeMajistre, R., Frisch, P. C., Heerikhuisen, J., Higdon, D. M., Janzen, P., Larsen, B. A., Livadiotis, G., McComas, D. J., Möbius, E., Reese, C. S., Reisenfeld, D. B., Schwadron, N. A., and Zirnstein, E. J.: Circularity of the Interstellar Boundary Explorer Ribbon of Enhanced Energetic Neutral Atom (ENA) Flux, Astrophys. J., 776, 30, doi:10.1088/0004637X/776/1/30, 2013.

Fuselier, S. A., Allegrini, F., Funsten, H. O., Ghielmetti, A. G., Heirtzler, D., Kucharek, H., Lennartsson, O. W., McComas, D. J., Möbius, E., Moore, T. E., Petrinec, S. M., Saul, L. A., Scheer, J. A., Schwadron, N., and Wurz, P.: Width and Variation of the ENA Flux Ribbon Observed by the Interstellar Boundary Explorer, Science, 326, 962-964, doi:10.1126/science.1180981, 2009.

Gry, C. and Jenkins, E. B.: A new perspective on the interstellar cloud surrounding the Sun from UV absorption line results, J. Phys. Conf. Ser., 577, 012012, doi:10.1088/17426596/577/1/012012, 2015.

Hébrard, G., Mallouris, C., Ferlet, R., Koester, D., Lemoine, M., Vidal-Madjar, A., and York, D.: Ultraviolet observations of Sirius A and Sirius B with HST-GHRS; An interstellar cloud with a possible low deuterium abundance, Astron. Astrophys., 350, 643-658, 1999.

Heerikhuisen, J., Pogorelov, N. V., Zank, G. P., Crew, G. B., Frisch, P. C., Funsten, H. O., Janzen, P. H., McComas, D. J., Reisenfeld, D. B., and Schwadron, N. A.: Pick-Up Ions in the Outer Heliosheath: A Possible Mechanism for the Interstellar Boundary EXplorer Ribbon, Astrophys. J. Lett., 708, L126, doi:10.1088/2041-8205/708/2/L126, 2010.

Heerikhuisen, J., Zirnstein, E. J., Funsten, H. O., Pogorelov, N. V., and Zank, G. P.: The Effect of New Interstellar Medium Parameters on the Heliosphere and Energetic Neutral Atoms from the Interstellar Boundary, Astrophys. J., 784, 73, doi:10.1088/0004637X/784/1/73, 2014.

Jokipii, J. R.: Our Interstellar Neighborhood, Science, 307, 1424 1425, doi:10.1126/science.1109701, 2005.

Jokipii, J. R.: A Local Wiggle in the Turbulent Interstellar Magnetic Field, Science, 316, 839-840, doi:10.1126/science.1141628, 2007.

Lallement, R., Bertin, P., Ferlet, R., Vidal-Madjar, A., and Bertaux, J. L.: GHRS observations of Sirius-A I. Interstellar clouds toward Sirius and Local Cloud ionization, Astron. Astrophys., 286, 898908, 1994.

Leonard, T. W., Möbius, E., Bzowski, M., Fuselier, S. A., Heirtzler, D., Kubiak, M. A., Kucharek, H., Lee, M. A., McComas, D. J., Schwadron, N. A., and Wurz, P.: Revisiting the ISN Flow Parameters, Using a Variable IBEX Pointing Strategy, Astrophys. J., 804, 42, doi:10.1088/0004-637X/804/1/42, 2015.

Linsky, J. L., Rickett, B. J., and Redfield, S.: The Origin of Radio Scintillation in the Local Interstellar Medium, Astrophys. J., 675, 413, doi:10.1086/526420, 2008.

McComas, D. J., Allegrini, F., Bochsler, P., Bzowski, M., Christian, E. R., Crew, G. B., DeMajistre, R., Fahr, H., Fichtner, H., Frisch, P. C., Funsten, H. O., Fuselier, S. A., Gloeckler, G., Gruntman, M., Heerikhuisen, J., Izmodenov, V., Janzen, P., Knappenberger, P., Krimigis, S., Kucharek, H., Lee, M., Livadiotis, G., Livi, S., MacDowall, R. J., Mitchell, D., Möbius, E., Moore, T., Pogorelov, N. V., Reisenfeld, D., Roelof, E., Saul, L., Schwadron, N. A., Valek, P. W., Vanderspek, R., Wurz, P., and Zank, G. P.: Global Observations of the Interstellar Interaction from the Interstellar Boundary Explorer (IBEX), Science, 326, 959-962, doi:10.1126/science.1180906, 2009a.

McComas, D. J., Allegrini, F., Bochsler, P., Bzowski, M., Collier, M., Fahr, H., Fichtner, H., Frisch, P., Funsten, H. O., Fuselier, S. A., Gloeckler, G., Gruntman, M., Izmodenov, V., Knappenberger, P., Lee, M., Livi, S., Mitchell, D., Möbius, E., Moore, T., Pope, S., Reisenfeld, D., Roelof, E., Scherrer, J., Schwadron, N., Tyler, R., Wieser, M., Witte, M., Wurz, P., and Zank, G.: IBEX-Interstellar Boundary Explorer, Space Sci. Rev., 146, 11 33, 2009b. 
McComas, D. J., Funsten, H. O., Fuselier, S. A., Lewis, W. S., Möbius, E., and Schwadron, N. A.: IBEX observations of heliospheric energetic neutral atoms: Current understanding and future directions, Geophys. Res. Lett., 38, L18101, doi:10.1029/2011GL048763, 2011.

McComas, D. J., Bzowski, M., Galli, A., Katushkina, O. A., Kucharek, H., Lee, M., Moebius, E., Park, J., Rodriguez, D., Schwadron, N., Sokól, J. M., Swaczyna, P., and Wood, B.: Local Interstellar Medium: Six Years of Direct Sampling by the Interstellar Boundary Explor, Astrophys. J. Suppl., in press, 2015a.

McComas, D. J., Bzowski, M., Frisch, P., Fuselier, S. A., Kubiak, M. A., Kucharek, H., Leonard, T., Möbius, E., Schwadron, N. A., Sokół, J. M., Swaczyna, P., and Witte, M.: Warmer Local Interstellar Medium: A Possible Resolution of the UlyssesIBEX Enigma, Astrophys. J., 801, 28, doi:10.1088/0004637X/801/1/28, 2015b.

Möbius, E., Bochsler, P., Bzowski, M., Crew, G. B., Funsten, H. O., Fuselier, S. A., Ghielmetti, A., Heirtzler, D., Izmodenov, V. V., Kubiak, M., Kucharek, H., Lee, M. A., Leonard, T., McComas, D. J., Petersen, L., Saul, L., Scheer, J. A., Schwadron, N., Witte, M., and Wurz, P.: Direct Observations of Interstellar H, He, and O by the Interstellar Boundary Explorer, Science, 326, 969-971, doi:10.1126/science.1180971, 2009.

Möbius, E., Bzowski, M., Frisch, P. C., Fuselier, S. A., Heirtzler, D., Kubiak, M. A., Kucharek, H., Lee, M. A., Leonard, T., McComas, D. J., Schwadron, N. A., Sokół, J. M., and Wurz, P.: Interstellar Flow and Temperature Determination with IBEX: Robustness and Sensitivity to Systematic Effects, Astrophys. J. Suppl. S., in press, 2015.

Pogorelov, N. V., Suess, S. T., Borovikov, S. N., Ebert, R. W., McComas, D. J., and Zank, G. P.: Three-dimensional Features of the Outer Heliosphere due to Coupling between the Interstellar and Interplanetary Magnetic Fields. IV. Solar Cycle Model Based on Ulysses Observations, Astrophys. J., 772, 2, doi:10.1088/0004637X/772/1/2, 2013.

Ptuskin, V. S., Moskalenko, I. V., Jones, F. C., Strong, A. W., and Zirakashvili, V. N.: Dissipation of Magnetohydrodynamic Waves on Energetic Particles: Impact on Interstellar Turbulence and Cosmic-Ray Transport, Astrophys. J., 642, 902-916, 2006.

Ratkiewicz, R., Strumik, M., and Grygorczuk, J.: The Effects of Local Interstellar Magnetic Field on Energetic Neutral Atom Sky Maps, Astrophys. J., 756, 3, doi:10.1088/0004-637X/756/1/3, 2012.

Redfield, S. and Linsky, J. L.: The Structure of the Local Interstellar Medium. IV. Dynamics, Morphology, Physical Properties, and Implications of Cloud-Cloud Interactions, Astrophys. J., 673, 283, doi:10.1086/524002, 2008.

Röken, C., Kleimann, J., and Fichtner, H.: An Exact Analytical Solution for the Interstellar Magnetic Field in the Vicinity of the Heliosphere, Astrophys. J., 805, 173, doi:10.1088/0004637X/805/2/173, 2015.
Schönrich, R., Binney, J., and Dehnen, W.: Local kinematics and the local standard of rest, Mon. Not. R. Astron. Soc., 403, 18291833, doi:10.1111/j.1365-2966.2010.16253.x, 2010.

Schwadron, N. A. and McComas, D. J.: Spatial Retention of Ions Producing the IBEX Ribbon, Astrophys. J., 764, 92, doi:10.1088/0004-637X/764/1/92, 2013.

Schwadron, N. A., Bzowski, M., Crew, G. B., Gruntman, M., Fahr, H., Fichtner, H., Frisch, P. C., Funsten, H. O., Fuselier, S., Heerikhuisen, J., Izmodenov, V., Kucharek, H., Lee, M., Livadiotis, G., McComas, D. J., Moebius, E., Moore, T., Mukherjee, J., Pogorelov, N. V., Prested, C., Reisenfeld, D., Roelof, E., and Zank, G. P.: Comparison of Interstellar Boundary Explorer Observations with 3D Global Heliospheric Models, Science, 326, 966-968, doi:10.1126/science.1180986, 2009.

Schwadron, N. A., Allegrini, F., Bzowski, M., Christian, E. R., Crew, G. B., Dayeh, M., DeMajistre, R., Frisch, P., Funsten, H. O., Fuselier, S. A., Goodrich, K., Gruntman, M., Janzen, P., Kucharek, H., Livadiotis, G., McComas, D. J., Moebius, E., Prested, C., Reisenfeld, D., Reno, M., Roelof, E., Siegel, J., and Vanderspek, R.: Separation of the Interstellar Boundary Explorer Ribbon from Globally Distributed Energetic Neutral Atom Flux, Astrophys. J., 731, 56, doi:10.1088/0004-637X/731/1/56, 2011.

Schwadron, N. A., Adams, F. C., Christian, E. R., Desiati, P., Frisch, P., Funsten, H. O., Jokipii, J. R., McComas, D. J., Moebius, E., and Zank, G. P.: Global Anisotropies in TeV Cosmic Rays Related to the Sun's Local Galactic Environment from IBEX, Science, 343, 988-990, doi:10.1126/science.1245026, 2014.

Schwadron, N. A., Moebius, E., Leonard, T., Fuselier, S. A., McComas, D. J., Heirtzler, D., Kucharek, H., Rahmanifard, F., Bzowski, M., Kubiak, M. A., Sokół, J., Swaczyna, P., and Frisch, P.: Determination of Interstellar He Parameters using 5 years of data from the Interstellar Boundary Explorer - beyond closed form approximations, Astrophys. J. Suppl. S., in review, 2015.

Shalchi, A., Büsching, I., Lazarian, A., and Schlickeiser, R.: Influence of Turbulence Dissipation Effects on the Propagation of Low-energy Cosmic Rays in the Galaxy, Astrophys. J., 725, 2110, doi:10.1088/0004-637X/725/2/2110, 2010.

Slavin, J. D. and Frisch, P. C.: The boundary conditions of the heliosphere: photoionization models constrained by interstellar and in situ data, Astron. Astrophys., 491, 53-68, 2008.

Wolleben, M.: A New Model for the Loop I (North Polar Spur) Region, Astrophys. J., 664, 349-356, 2007.

Wood, B. E., Linsky, J. L., and Zank, G. P.: Heliospheric, Astrospheric, and Interstellar Ly $\mathrm{L}_{\alpha}$ Absorption toward 36 Ophiuchi, Astrophys. J., 537, 304-311, 2000.

Wood, B. E., Müller, H.-R., and Witte, M.: Revisiting Ulysses Observations of Interstellar Helium, Astrophys. J., 801, 62, doi:10.1088/0004-637X/801/1/62, 2015. 